\title{
Image Reconstruction in Spectrally Resolved 3D Bioluminescence Tomography using the Adjoint Theorem
}

\author{
Hamid Dehghani ${ }^{1}$, Scott C. Davis ${ }^{2}$, and Brian W. Pogue ${ }^{2}$ \\ ${ }^{I}$ School of Physics, University of Exeter, UK \\ ${ }^{2}$ Thayer School of Engineering, Dartmouth College, Hanover, NH 03755, USA \\ email:h.dehghani@exeter.ac.uk,Tel:+441392 264177,Fax:+441392261111
}

\begin{abstract}
Spectrally-resolved BioLuminescence optical Tomography (sBLT) is used to recover images of Luciferase activity within a 3D model using multi-wavelength emission data from internal bioluminescence sources. The Adjoint theorem for 3D image reconstruction is presented and validated using experimental data with a complete computation time of 5 minutes.

(c)2006 Optical Society of America

OCIS codes: (170.6960) Tomography; (100.3010) Image reconstruction techniques; (100.3190) Inverse problems
\end{abstract}

\section{Introduction}

The development and widespread implementation of bioluminescence imaging has resulted in cancer research undergoing a significant advance in the ability of investigators to determine cell growth in vivo [1]. Tumor metastases studies have been completed with luciferase imaging, showing tumor cell invasion, which otherwise could not be observed [2]. As these imaging and measuring tools progress, it is important to have improved imaging and image reconstruction methods at appropriate computational speed to accurately visualize and quantify the bioluminescence signals in vivo in order to gain fundamental insight into tumor growth, regression, immune response and cellular response to therapy. Specifically it is essential to develop 3D imaging methods to combine information from multiple imaging technologies, and further, to use the information from different sources to generate improved and increased understanding of the processes under investigation.

Recent interest in modeling and reconstruction algorithms for BLT has increased [3-5] and led to the general consensus that non-spectrally resolved intensity-based BLT results in a non-unique problem[6]. However, the light emitted from firefly Luciferase is widely distributed over the band of wavelengths from $500 \mathrm{~nm}$ to $650 \mathrm{~nm}$ and above. When not attenuated, it produces a peak emission near $560 \mathrm{~nm}$, but when detected from within an animal appears to have a peak closer to $600 \mathrm{~nm}$ with measurable emissions as much as $50 \mathrm{~nm}$ above and below this peak [1]. Using a spectrometer it is possible to measure the emission at the surface of the tissue in discrete steps of $10 \mathrm{~nm}$ ranging from $550 \mathrm{~nm}$ to $650 \mathrm{~nm}$, although for deeper sources strong optical absorption of tissue at the lower wavelengths might preclude accurate measurements with adequate signal to noise.

To date, all reconstruction algorithms have either used a-priori information to constrain the solution and keep the computation time low [5], or they have used analytical solution which limits the problem to homogenous tissue [4]. This paper demonstrates the development of a 3D algorithm used for multi-wavelength 3D BLT image reconstruction using experimental data. The Adjoint theorem, similar to those seen Diffuse Optical Tomography (DOT) [7] and Fluorescence DOT [8] are presented, showing that accurate 3D images can be obtained using a fast linear reconstruction algorithm without the use of a-priori information.

\section{Method}

The mathematical basis for sBLT is the Boltzmann transport equation, a physical model for light propagation in tissue. Under the assumption that scattering dominates absorption for red light in tissue, the Boltzmann transport equation can be simplified to the diffusion approximation, which for a continuous light source is given by:

$$
-\nabla \cdot D(\boldsymbol{r}) \nabla \Phi(\boldsymbol{r})+\mu_{a} \Phi(\boldsymbol{r})=B(\boldsymbol{r})
$$

where $B(r)$ is an internal bioluminescence source, $\Phi(r)$ is the photon fluence rate at position $r, D=1 / 3\left(\mu_{a}+\mu_{s}{ }^{\prime}\right)$ is the diffusion coefficient, $\mu_{a}$ is the optical absorption coefficient and $\mu_{s}$ ' is the reduced scatter coefficient. The fluence rate data can be represented by an operator, which is linear in terms of the bioluminescence source. Assuming that absorption and scatter are known from separate measurements and reconstructions [9], the BLT image reconstruction problem is posed as a solution to the minimization:

$$
\hat{\chi}=\arg \min _{\mu_{a}, \kappa}\|(y-F(B(r)))\|
$$

where $y$ are the measured data, $F(B(r))$ are the data calculated using equation (1) given an estimate of the internal bioluminescence distribution model, and \|..\| is the weighted L2-norm, representing the square root of the sum of the 


\section{BMC4.pdf}

squared elements. When no prior information about source location is available, the problem is inherently more difficult, since both the source strength and its spatial distribution may vary. However, the photon fluence rate, which is the measured observable, is linear in $B(r)$; therefore, a practical approach to solving this problem constructs the image of the bioluminescence source as a sum of basis distributions whose weights are estimated from the measured response. Taking advantage of the linearity of the model it is possible to create a set of independent basis solutions for the source, $B=\sum_{i=1}^{N} a_{i} b_{i}$, where the coefficients $a_{i}$ are the weight functions for multiple unit sources $b_{i}$ at each node $i$ in the 3D model containing a total number of nodes $N$, which in matrix form is given as, $B=a b$. Substituting this matrix expression into equation 2 and solving for $a$, in a least square manner, results in a single step linear expression, $a=W^{T}\left(W W^{T}+\lambda I\right)^{-1} y$, where $W$ is a matrix containing the solution of equation (1) for all possible source positions $N$ and y is the measured surface flux. Here, $\lambda$ is a regularization parameter and $I$ is the identity matrix. Although the Hessian matrix $W W^{T}$ is invertible, the use of $\lambda$ becomes necessary in the presence of noise in the data. In this work, $\lambda=0.001 \%$ of the maximum of the diagonal of the Hessian is used. Instead of using data from a single wavelength, multi-wavelength sBLT combines data-sets that are measured from the same domain containing the same bioluminescence distribution, over a range of usable wavelengths such that $a=\hat{W}^{T}\left(\hat{W} \hat{W}^{T}+\lambda I\right)^{-1} \hat{y}$, where $\hat{W}=\left[W_{\lambda 1} ; W_{\lambda 2} ; W_{\lambda 3} ; \ldots ; W_{\lambda n}\right]$ are the weight matrices of all $n$ of cascaded wavelengths and $\hat{y}=\left[y_{\lambda 1} ; y_{\lambda 2} ; y_{\lambda 3}\right.$; ...; $\left.y_{\lambda n}\right]$ is the corresponding measured surface data for each wavelength. The solution $a$ is a vector corresponding to the number of unknowns that define the bioluminescence source distribution.

The weight matrix is conventionally calculated using the direct, or so called 'perturbation' method, whereby each point within the model is perturbed by a small amount and the resulting data at the detectors calculated. Using this method, for a finite element mesh of 10,000 nodes, therefore 10,000 forward solutions are needed. The Adjoint method, as derived for DOT [7] and Fluorescence DOT [8] can also be used for BLT which simply takes into account the reciprocity properties of equation (1) [10]. That is the solution at a detector due to a given point within the model is equal to the solution at the same point if the detector was the source. Using this method, for a finite element mesh of 10,000 nodes, and 16 detector point only 16 forward solutions are needed.

In order to demonstrate the validity of the Adjoint method using experimental data, measurements were recorded for a single bioluminescence source placed within an otherwise uniform cylindrical phantom of $50.8 \mathrm{~mm}$ diameter, Figure $1(\mathrm{a})$. The phantom was filled with a solution of $0.2 \%$ Intralipid and $0.2 \%$ oxygenated blood. The bioluminescent source was a 50:50 mixture of 165 microMolar ATP solution with Luciferase reagent solution solubolized in $1 \mathrm{ml}$ distilled water (CLS II Kit, Roche Diagnostics Inc.). The solution was held in a plastic cylinder of diameter $5 \mathrm{~mm}$, placed $7 \mathrm{~mm}$ inside the edge at the 3 o'clock position. Sixteen equally spaced measurements around the periphery were collected sequentially using a $1 \mathrm{~mm}$ silica fiber bundle leading to a single line of 200 micron fibers entering a spectrometer (Acton SpectraPro 2300i spectrometer with $300 \mathrm{l} / \mathrm{mm}$ grating blazed at $750 \mathrm{~nm}$ wavelength) coupled to a cooled CCD (Princeton Instruments Spec-10 XTE CCD). Integration time was 15 minutes per sample, and a fresh sample was used for each detector location to avoid the effect of source decay. The measured spectrum of the bioluminescence source was normalized using an un-attenuated emission spectrum from a dilute sample.

(a)

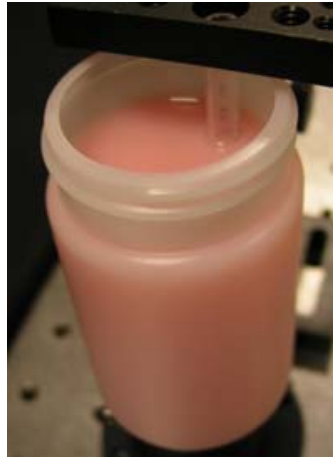

(b)

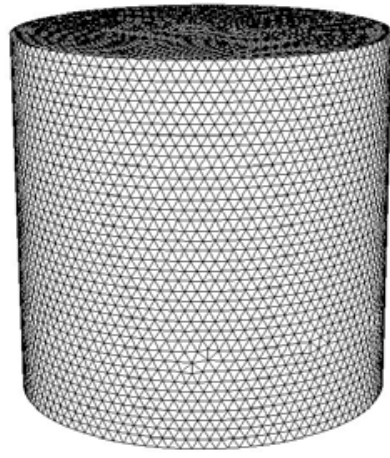

Figure 1. (a) Experimental setup of data collection, and (b) 3D cylindrical mesh used for image reconstruction.

Using a 3D cylindrical mesh, Figure 1(b), containing 27,999 nodes corresponding to 145,835 linear tetrahedral elements a 3D image was reconstructed using the algorithm defined above. This image was based on 5 wavelength intervals of $10 \mathrm{~nm}$, ranging from $600-650 \mathrm{~nm}$. Over this range the absorption coefficient varied from 0.0009 to $0.0043 \mathrm{~mm}-1$ and the reduced scattering from 0.34 to $0.4 \mathrm{~mm}-1$ across the wavelengths used. 


\section{BMC4.pdf}

\section{Results}

As evident in Figure 2, the 3D reconstructed image using multi-wavelength data and the Adjoint theorem has accurately estimated the size and location of the internal bioluminescence source at approximately $7.5 \mathrm{~mm}$ from the surface with a Full Width Half Maximum of $8 \mathrm{~mm}$, despite the relatively coarse spatial sampling of the emission. Instrumentation improvements, such as more boundary data and improved signal quality are expected to improve the quality of images. The time for the calculation of the weight matrix for the model was approximately 1 minutes per wavelength (using a dual Xeon 3.7GHz, 4GB RAM), giving a total reconstruction time of approximately 5 minutes including matrix inversion.
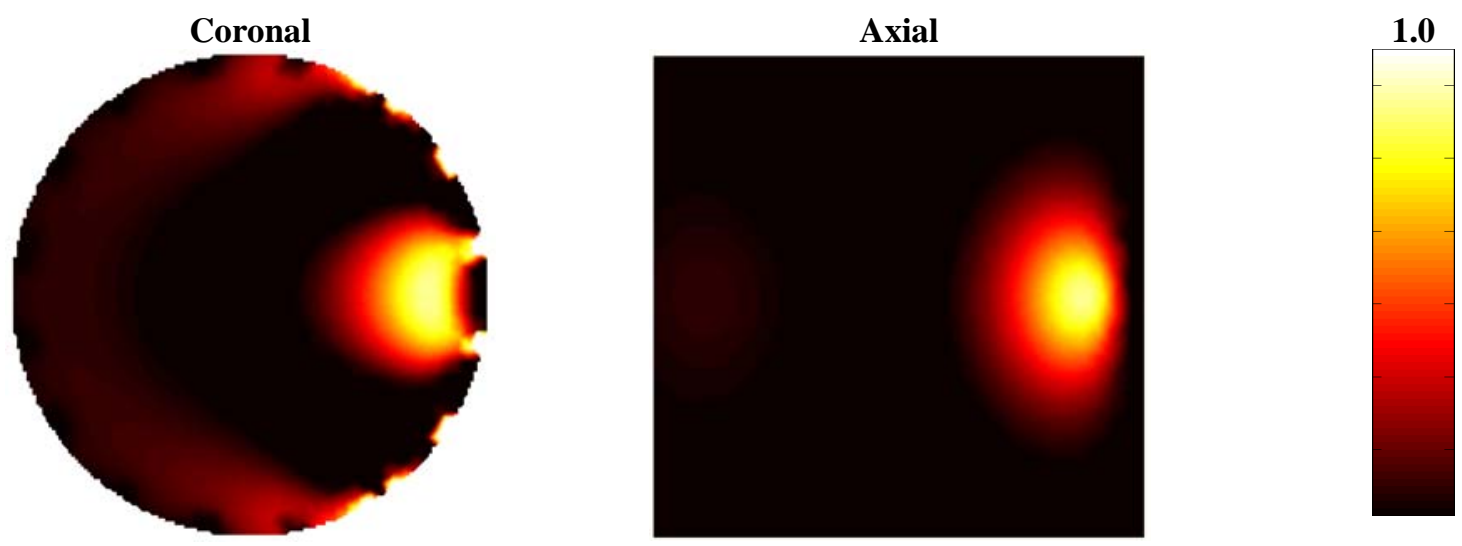

Figure 2. 3D reconstructed image of Bioluminescence using the Adjoint Theorem.

\section{Discussion}

In this paper a modeling and linear single step image reconstruction algorithm for sBLT is presented together with the Adjoint theorem which demonstrates computationally fast BLT image recovery from 3D multi-wavelength data. Multi-wavelength emission provides a means of estimating the depth of an object, due to the wavelength dependent attenuation of tissue. Using the theory presented here, it has been demonstrated that without the use of a-priori information about the location of the bioluminescence source accurate 3D images can be obtained at relatively fast computation time.

\section{Acknowledgements}

This work has been sponsored by EPSRC, UK and the National Cancer Institute through grants RO1CA109558 and P01CA80139.

\section{References}

1. Contag, C.H., Bachmann, M. H., Adavnces in in vivo bioluminescence imaging of gene expression. Annual Review of Biomedical Engineering, 2002. 4: p. 235-260.

2. $\quad$ Contag, C.H., Ross, B. D., It's not just about anatomy: In vivo bioluminescence imaging as an eyepiece into biology. J Magn Reson Imaging, 2002. 16: p. 378-387.

3. Dehghani, H., Davis, S. C., Jiang, S., Pogue, B. W., Paulsen, K. D., and Patterson, M. S., Spectrally-Resolved Bioluminescence Optical Tomography. Optics letters, 2006. 31(3): p. 365-367.

4. $\quad$ Kuo, C., Coquoz, O., Troy, T. L., Xu, H., and Rice, B. W., Three-dimensional reconstruction of in vivo bioluminescent sources based on multispectral imaging. JBO, 2007. 12: p. 24007.

5. Wang, G., Cong, W., Durairaj, K., Qian, X., Shen, H., Sinn, P., Hoffman, H., McLennan, G., and Henry, M., In vivo mouse studies with bioluminescence tomography. Optics Express, 2006. 14(17): p. 7801-7809.

6. $\quad$ Wang, G., Li, Y., Jiang, M., Uniqueness theorems in bioluminescence tomography. Med. Phys., 2004. 31(8): p. 2289-2299.

7. $\quad$ Arridge, S.R., Optical tomography in medical imaging. Inverse Problems, 1999. 15(2): p. R41-R93.

8. Milstein, A.B., Oh, S., Webb, K. J., Bouman, C. A., Zhanng, Q., Boas, D. A. and Millane, R. P., Fluorescence optical diffusion tomography. Applied Optics, 2003. 42(16): p. 3081-3094.

9. $\quad$ Srinivasan, S., Pogue, B. W., Jiang, S., Dehghani, H., and Paulsen, K. D., Spectrally Constrained Chromophore and Scattering NIR Tomography Provides Quantitative and Robust Reconstruction. Appl. Opt., 2005. 44(10): p. 1858-1869.

10. Chew, W.C., Waves and Fields in Inhomogeneous Media. 1990: Van Nostrand Reinhold, New York. 